\title{
Price carbon - I will if you will
}

\author{
A common goal that is in everyone's self-interest is needed for countries to sign up to a strong climate \\ agreement at Paris, say David MacKay and colleagues.
}

Climate-change negotiations in Paris this December will adopt a 'pledge and review' approach to cutting global carbon emissions. Countries will promise to reduce their emissions by amounts that will be reviewed and revised later. The narrative is that open review will "enable an upward spiral of ambition over time" ${ }^{1}$. Unfortunately, history and the science of cooperation predict quite the opposite will happen.

Climate is a serious challenge because the atmosphere gives a free ride to countries that emit. If some nations sit back and rely on others' cooperative efforts, the incentives for anyone to act are weakened. The comparable review of Kyoto's phase one, at the 2012 Doha conference, resulted in Japan, Russia, Canada, and New Zealand leaving the agreement, frustrating those who kept their promises.

Success requires a common commitment, not a patchwork of individual commitments. The negotiation process must be designed to redirect self-interest to promote cooperation by using a common commitment to assure players that others will match their efforts and not free-ride. Simple game theory shows that this may be achieved when interests are aligned. "I will if you will" is the most robust pattern of cooperation seen in laboratory, field and theoretical studies of free-rider situations, and is consistently found to stabilize higher levels of cooperation ${ }^{2}$.

A global carbon price, so far excluded from consideration, would be the ideal basis for a flexible common commitment in our view. A single price is easy to agree on, relatively fair, easy to handle, less vulnerable to gaming than global cap-and-trade, and consistent with climate policies already in place, such as fossil-fuel taxes and cap-and-trade.

Only a common commitment can lead to a strong treaty that makes enforcement effective.

\section{Pledges fail}

Forty years of empirical and theoretical literature on cooperation confirms that individual commitments, such as pledged national contributions, do not deliver strong collective action. Cooperators will find that defectors take advantage of them. As a result, initial ambition declines, when reviewing reveals that others are freeriding $^{3}$. For example, without a common commitment that manages individual contributions, dishes often stack up in the sinks of offices and shared apartments. But with an explicit common commitment governing grasslands in the Alps, villagers have successfully managed the same commons for hundreds of years. ${ }^{4}$

Imagine that you and nine other self-interested players (representing countries) play a game. Each player has $\$ 10$, of which they simultaneously pledge some part to the common pot. A referee makes sure they honor their pledges. Every dollar (for $\mathrm{CO}_{2}$ abatement) placed in the pot will be doubled (by climate benefits) and distributed evenly to all players. So putting a dollar in the pot will return $20 c$ to each player.

First, in the 'individual commitment' game, pledges are independent of those of others. This is the classic publicgoods game, in which the rational strategy for the narrowly self-interested is to contribute nothing, since this makes a player better off no matter what the others do. The result is the famous tragedy of the commons. Cooperation does not occur even though everyone would gain from it.

Second, consider the 'common commitment' game in which the same players commit to reciprocally match the contribution of others, but not more. More specifically, suppose the referee simply makes sure that all contribute the amount of the lowest submitted pledge. After enforcing this common commitment, the money is doubled and distributed evenly, exactly as before.

This changes everything. Pledging $\$ 0$ will mean simply keeping your $\$ 10$, while pledging $\$ 10$ could result in ending up with anything between $\$ 10$ and $\$ 20$, depending on what others pledge. So, since you cannot lose and could gain by pledging $\$ 10$, that's what you would do, even if you are completely selfish. So all pledge $\$ 10$, and the group's $\$ 100$ is doubled and all end up with the maximum amount of $\$ 20$. 
Because the common commitment protects against free-riding, selfish behavior has been changed from "contribute nothing" to "contribute everything"; the outcome from no cooperation to full cooperation.

The Kyoto negotiators initially did try to agree a common commitment expressed in terms of a formula for setting emissions caps for individual countries, but they failed to find an acceptable common formula. In the end each nation was asked to "submit their final numbers to the podium." Those numbers were simply inserted into the blank draft annex $\mathrm{B}^{5}$. The result was a patchwork of weak and unstable individual commitments. Similarly, in response to the Copenhagen accord which invited voluntary individual commitments, China pledged to do exactly what the US Department of Energy, a year earlier, had predicted it would do anyway, without cooperation, and India pledged half of the DOE prediction. Annual reviews did not cause them to increase their ambition.

Enforcement is widely thought to be the missing ingredient in the Kyoto Protocol and crucial for the success of a Paris agreement. This is half right. Both enforcement and a common commitment are required ${ }^{6}$. For example, if people chose their own road speed limits, there would be no use enforcing them, as everyone would drive at their desired speed. Instead, because it limits others as well as themselves, people agree to a common speed limit which is lower than almost everyone's individual limit. In other words, with individual commitments, there is nothing meaningful to enforce; whereas, enforcement strengthens a common commitment.

What could all countries commit to? National limits on the quantity of emissions will not work. Kyoto negotiators tried, and suggested at least 10 formulae to determine the reductions each nation should make, yet they could not agree. When attention turned to reducing emissions by a constant percentage relative to 1990 , individual commitments ranged from an $8 \%$ decrease to a $10 \%$ increase. And within the EU's $8 \%$ decrease, EUmember-states' commitments ranged from a decrease of $30 \%$ to an increase of $40 \%$. The US and developing countries accepted no commitments at all.

Kyoto's percent-emission-reduction concept failed because countries differ in many ways; for instance, some economies declined after 1990 and some grew. To be cautious, the developing countries must build in high growth aspirations, implying rapidly increasing caps. Rich countries oppose this, and so poor countries fear being saddled with caps that curb their growth. Instead they see it as fair to allocate emission permits on an equal per-capita basis. Because this would result in huge wealth transfers from rich to poor countries, such proposals are unacceptable to the rich ${ }^{7}$.

In fact, there is no longer any serious discussion of a common quantity commitment. The US government concurs that the approach will not work ${ }^{8}$. Developing countries still have not accepted it within the Kyoto framework. A common quantity commitment will not be discussed at COP 21 in Paris.

\section{Price carbon}

We, and others, propose an alternative: a global carbon price commitment ${ }^{9}$. Each country would commit to placing enough nationally-retained charges on their own use of fossil fuels to meet the common price commitment. The price level could be determined by voting-by a super-majority rule that would produce a coalition of the willing. Note that a uniform price is already widely accepted as the most cost-effective way to curb emissions.

Carbon pricing is flexible, allowing fossil taxes, cap-and-trade, hybrid schemes and other national policies to be used (unlike a global carbon tax). All that is required of a country is that its average carbon price-carbon revenue divided by carbon emissions-be at least as high as the global carbon price.

Unlike global cap-and-trade, carbon pricing allows countries to keep all carbon revenues, thus eliminating the risk of needing to buy expensive credits from a rival country. A country can hold its total taxes constant by a green tax-shift-reducing taxes on good things such as employment by charging for pollution. Shifting taxes from good things to bad things could mean there is no net social cost to pricing carbon, even before counting climate benefits ${ }^{10}$.

Of course a global price does not automatically result in acceptable burden sharing. A 'Green Climate Fund' will be needed to transfer funds from rich to poor countries. To minimize disputes over fairness, the objective of 
climate-fund transfers should be to maximize the global price of carbon. This can be implemented in a way that encourages rich countries to be generous and poor countries to vote for a higher global carbon price ${ }^{11}$ for example, by making all climate-fund payments proportional to the agreed-upon carbon price.

After decades of failure, a fresh approach is needed-one that is guided by the science of cooperation. A common price commitment would harness self-interest by aligning it with the common good. Nothing could be more fundamental.

\title{
Authors:
}

David MacKay, Peter Cramton, Axel Ockenfels, and Steven Stoft ${ }^{12}$

\author{
David J.C. MacKay FRS \\ Department of Engineering \\ University of Cambridge \\ Trumpington Street, \\ Cambridge CB2 1PZ, UK \\ Peter Cramton \\ Department of Economics \\ University of Maryland \\ College Park, MD 20742 \\ USA \\ Axel Ockenfels \\ Department of Economics \\ Center for Social and Economic Behavior \\ University of Cologne \\ D-50923 Cologne \\ Germany
}

Steven Stoft

2910 Elmwood Ct.

Berkeley, CA 94705

USA

\footnotetext{
${ }^{1}$ Ad Hoc Working Group on the Durban Platform, ADP.2014.6.NonPaper, 7 July 2014.

2 E. Ostrom (1990), Governing the Commons: The Evolution of Institutions for Collective Action, Cambridge University Press; D. Fudenberg and J. Tirole (1991) Game Theory, Cambridge, MA: MIT Press; S. Bowles and H. Gintis (2013), A Cooperative Species: Human Reciprocity and Its Evolution, Princeton University Press; Kraft-Todd et al. (2015), "Promoting Cooperation in the Field", Current Opinion in Behavioral Sciences, 3:96-101.

${ }^{3}$ See, e.g., J. Ledyard (1995), "Public goods: A survey of experimental research", in J.H. Kagel and A.E. Roth, eds., The Handbook of Experimental Economics, NJ: Princeton University Press, 111-194; E. Ostrom (1998), A Behavioral Approach to the Rational Choice Theory of Collective Action: Presidential Address, American Political Science Review, 92(1), 1-22; D.G. Rand and M. Nowak (2013), "Human Cooperation", Trends in Cognitive Sciences, 17(8), 413-425, and our references in footnote 2.

${ }^{4}$ E. Ostrom (1990), Governing the Commons: The Evolution of Institutions for Collective Action, Cambridge University Press.

${ }^{5}$ J. Depledge (2000), The Origins of the Kyoto Protocol, prepared under contract to the UNFCCC, FCCC/TP/2000/2.

${ }^{6}$ In our example individual commitments fail even though the referee can fully enforce all pledges. Also, when Iceland made its individual commitment to the Kyoto Protocol it said it thought it could not keep it. Had Kyoto adopted strong enforcement, Iceland would have weakened its pledge rather than risk the nearly inevitable punishment.

7 E.g., J. Stiglitz (2006), "Saving the Planet", chapter 26 in Making Globalization Work, Norton.

${ }^{8}$ In its 11 March 2013 submission, the US stated, "It is hard to imagine agreement on any formula or criteria for imposition of contributions, as this would get into the most controversial issues".
} 
${ }^{9}$ See, e.g., M. Weitzman (2015), “Internalizing the Climate Externality: Can a Uniform Price Commitment Help?”, and J. Stiglitz (2015), "Overcoming the Copenhagen Failure with Flexible Commitments", as well as other papers published in the "Symposium on International Climate Negotiations" in Economics of Energy \& Environmental Policy, 2015, 4:2, edited by Cramton, Ockenfels and Stoft. All papers in the symposium, plus additional relevant material, can be accessed at carbon-price.com.

${ }^{10}$ D.W. Pearce (1991), "The Role of Carbon Taxes in Adjusting to Global Warming." Economic Journal 101:938-948; A.L. Bovenberg (1999): "Green Tax Reforms and the Double Dividend: An Updated Reader's Guide", International Tax and Public Finance 6, 421443; see for a related argument M. Franks, O. Edenhofer, and K. Lessmann (2015), "Why Finance Ministers Favor Carbon Taxes, Even if They Do Not Take Climate Change into Account", Harvard Project on Climate Agreements, Discussion Paper 2015-67.

${ }^{11}$ P. Cramton and S. Stoft (2012), "Global Climate Games: How Pricing and a Green Fund Foster Cooperation", Economics of Energy \& Environmental Policy, 1:2.

12 MacKay: Cambridge University Engineering Department, Cramton: University of Maryland, European University Institute, and University of Cologne, Ockenfels: University of Cologne, Stoft: Berkeley and author for correspondence (sstoft@gmail.com). Financial support of the German Research Foundation (DFG) through the Research Unit "Design \& Behavior" (FOR 1371) is gratefully acknowledged. 